\title{
Minimal deviation adenocarcinoma ("adenoma malignum") of the cervix: A diagnostic dilemma
}

\author{
Dr. Pooja Banerjee ${ }^{1}$ Dr.Partha Sarathy Mitra ${ }^{2}$, Dr.Pradip Bhowmik ${ }^{3}$, \\ Dr.Prabodh S.Soreng ${ }^{4}$, Dr. Biswapati Mukherjee ${ }^{5}$, Dr.Subhendu Choudhury ${ }^{6}$ \\ 1,2,4Dept. obstetrics and gynaecology, medical college,Kolkata, India \\ ${ }^{3}$ Dept.cardiology, medical college, Kolkata, India
}

\begin{abstract}
Adenoma malignum is a rare variant of cervical adenocarcinoma. A woman of 26 years with complains of profuse mucoid discharge per vaginum on speculum examination per vaginum showed multiple cauliflowers like growth hanging within the introitus. Punch biopsy gave a report of endocervical glandular polyp with squamous metaplasia. On excision, the mass was found to arise from the cervix and external Os was separately identified. On histopathological examination the final diagnosis was adenoma malignum. Adenoma malignum of the uterine cervix is difficult to diagnose because of the deceptively benign appearance. To make a correct diagnosis, it is necessary to find a characteristic pathological feature such as multiple irregular lobulations of distorted glands demonstrating a "hair-pin" shape. Despite the presence of well-differentiated histopathological features, the prognosis of adenoma malignum is known to be poor because of early dissemination into the peritoneal cavity and early distant metastasis.
\end{abstract}

Key word: Adenoma malignum (AM)

\section{Introduction}

Adenoma malignum or minimal deviation adenocarcinoma of the cervix is a rare variant of cervical adenocarcinoma with poor prognosis ${ }^{1}$. Most of the cases are diagnosed not by the attending gynecologist, rather by the pathologist while examining the pathology specimen. Preoperative diagnosis is difficult as cervical cytology, colposcopy guided biopsy and serum markers such as carcino embryonic antigen (CEA) are not very sensitive for detecting this variety of cervical cancer ${ }^{2,3}$. A preoperative punch biopsy fails to confirm a diagnosis of MDA in most cases ${ }^{4}$. Imaging study such as pulsed power Doppler and Magnetic Resonance Imaging are upcoming investigation modalities used for differentiating adenoma malignum from other cystic lesions of cevix $^{5-7}$. Currently genetic markers linked to chromosome $19 \mathrm{pl} 3.3$ are found to be associated with the Sporadic Form of adenoma malignum of the uterine cervix ${ }^{7}$. Here we present a case of adenoma malignum for its peculiarity of presentation.

\section{Case report}

The present case is a woman of 26 years age married for 9 years $\mathrm{P} 0+1+1+0$ who came to the gynecology out patient department with complains of profuse mucoid discharge per vaginum with occasional spotting. She had a regular menstrual history. General examination findings were normal. On speculum examination per vaginum we saw multiple cauliflower like growth hanging within the introitus. Per vaginal palpation showed a growth occupying the vagina. Lateral vaginal walls were free while the cervical margins couldn't be felt.

Multiple possibilities came into our mind such as cervical cancer, a degenerated polyp or sub mucous fibroid, Sarcoma botryoides, uterine inversion, viral warts, vaginal growth and Hydatidiform mole. Smear from the growth was sent for bacteriological culture, Human papilloma virus antigen assay and a punch biopsy was sent for histopathological examination. Bacterial and virological studies were negative while punch biopsy gave a report of endocervical glandular polyp with squamous metaplasia.

We then performed an examination under anesthesia where we couldn't identify the external Os, uterus was normal size, anteverted and fornices were free. On Per rectal examination pouch of Douglas and parametrium were free. Then after inserting a Foley's catheter we attempted to excise the mass after applying successive haemostatic clamps (picture 1). On excision, the mass was found to arise from the cervix and external Os was separately identified(picture 2). On introduction of uterine sound uterine cavity was found to be of normal size.

After excision and histopathological examination the final diagnosis was adenoma malignum. The patient subsequently underwent Wertheim's hysterectomy followed by radiotherapy. She is disease free for two years after treatment completion. 


\section{Discussion}

As early as in 1896, Hellier reports a case of Adenoma Malignum Cysticum Cervicis Uteri as "a growth which sprang from the cervix and filled the vagina without involving it. Vagina was found to be filled with cauliflower-like growth, necrotic and foul. When this was scraped away it was found that the vaginal walls were not involved and that the uterus was quite mobile" a description very similar to the present case".

Subsequently Mckelvey and Goodlin in a case series described various presentations of Adenoma Malignum, red, granular, nodular cervix with the lesion invading the vagina; friable, cauliflower- shaped mass; cervical erosion; recurrence in cervical conisation stump or vault recurrence subsequent to hysterectomy ${ }^{10}$.

Adenoma malignum of the uterine cervix also referred to as the mucinous type of minimal deviation adenocarcinoma (mucinous MDA) of the uterine cervix, is a unique neoplasm that is difficult to diagnose because of the deceptively benign appearance of the tumor cells ${ }^{1}$. It is a subtype of mucinous adenocarcinoma of the uterine cervix, which represents about $3 \%$ of adenocarcinoma cases ${ }^{3}$. In its well-developed form, the malignancy of the tumor, particularly as judged from biopsy, is being missed by competent pathologists, leading to a possibility of being under treated. Some reports suggest cytological examination as a better aid to detect this tumor with an $83 \%$ success rate ${ }^{11}$. The common cytological features of mucinous MDA are (1) the frequent appearance of large sheets of cells with a honeycomb pattern on end view and a palisading arrangement at the periphery, (2) abnormal glandular cells with abundant cytoplasm showing slight to overt atypia in addition to benign-appearing glandular cells, and (3) slightly enlarged mucous cells with ovoid nuclei ${ }^{12-15}$. To make a correct diagnosis, it is necessary to find a characteristic pathological feature such as multiple irregular lobulations of distorted glands demonstrating a "hair-pin" shape ${ }^{4}$. However, the histopathological features of adenoma malignum are occasionally similar to pseudoneoplastic glandular lesions ${ }^{16,17}$. The MR findings of pseudoneoplastic lesions were almost identical to those of adenoma malignum, which suggested that the presence of cellular atypia or structural dysplasia in the pseudoneoplastic lesions did not significantly change MR findings 5,15 .

Mucinous MDA characteristically expresses gastric phenotypes. Immunostaining using the monoclonal antibody HIK1083, which is specific for gland mucous cell mucin of the stomach, facilitates the identification of tumor cells in tissue sections ${ }^{18}$. Despite the presence of well-differentiated histopathological features, the prognosis of adenoma malignum is known to be poor because of early dissemination into the peritoneal cavity and early distant metastasis. This is true of even the early lesions so that it has a bad prognostic outlook ${ }^{2-4}$. This is in contradistinction to the classical adenocarcinoma of the cervix which has shown a $50 \% 5$-year cure rate and is quite comparable in this respect to the squamous cell carcinomas.

AM Legend 1: mass seen during EUA

Figure Legend

AM Legend 2: excised mass

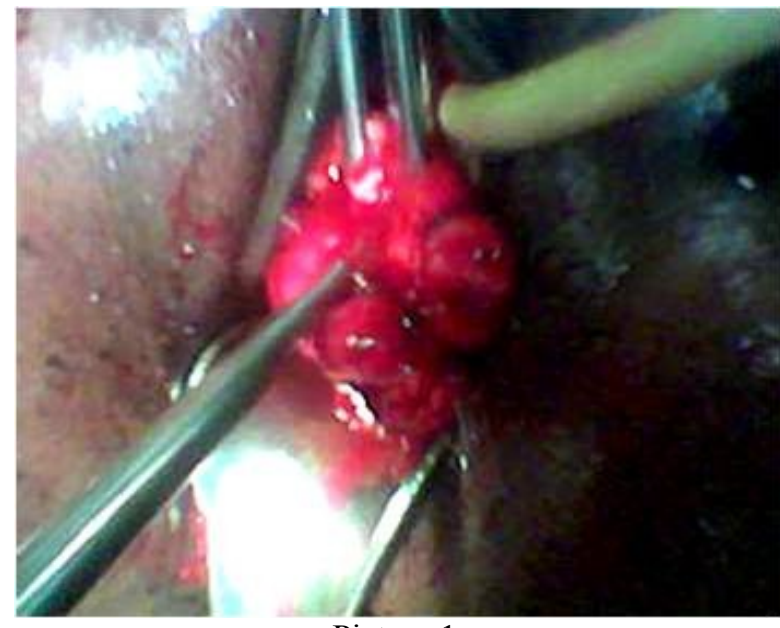

Picture 1 


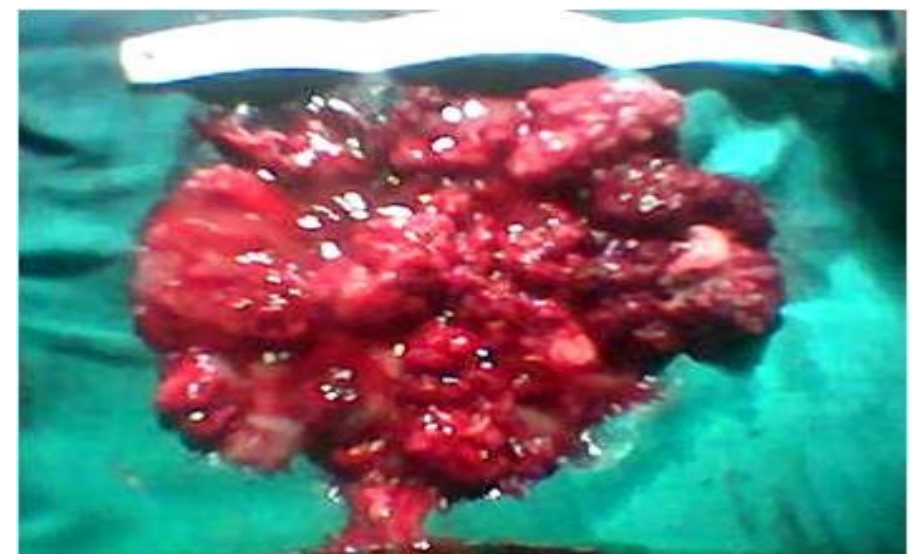

Picture 2

\section{References}

[1]. Silverberg SG and Hurt WG. Minimal deviation adenocarcinoma (adenoma malignum) of the cervix. Am. J. Obstet. Gynecol. 1975; 23:971-975

[2]. TA Steeper and MR Wick. Minimal Deviation Adenocarcinoma of the Uterine Cervix ("Adenoma Malignum ").An lmmunohistochemical Comparison with Microglandular Endocervical Hyperplasia and Conventional Endocervical Adenocarcinoma.Cancer (1986); 58:1131-1138.

[3]. Gilks CB, Young RH, Aguirre P, DeLellis RA, Scully RE .Adenoma malignum (minimal deviation adenocarcinoma) of the uterine cervix. A clinicopathological and immunohistochemical analysis of 26 cases. Am J Surg Pathol(1989);13(9):717-29.

[4]. Mulvany NJ, Monostori SJ. Adenoma malignum of the cervix: a reappraisal. Pathology (1997); 29: 17-20.

[5]. Sugiyama K and Takehara Y. MR findings of pseudoneoplastic lesions in the uterine cervix mimicking adenoma malignum. The British Journal of Radiology (2007); 80: 878-883.

[6]. Dui T, Yamashita Y, Yasunaga T, Fujiyoshi K, Tsunawaki A, Takahashi M et al. Adenoma malignum: MR imaging and pathologic study. Radiology (1997); 204:39-42.

[7]. Umesaki N, Nakai Y, Honda K, Kawamura N, KanaY.Power Doppler Findings of Adenoma malignum of Uterine Cervix.Gynecologic and Obstetric Investigation (1998); 45 (3): 213-216.

[8]. Lee JY, Dong SM, Kim HS, Kim SY, Na EY, Shin MS et al. A Distinct Region of Chromosome 19pl3.3 Associated with the Sporadic Form of Adenoma Malignum of the Uterine Cervix. Cancer Research (1998); 58: 1140-1143.

[9]. Hellier JB. Adenoma Malignum Cysticum Cervicis Uteri. Proc R Soc Med.(1910);3(Obstet Gynaecol Sect):100-3.

[10]. JL.Mckelvey and RR.Goodlin. Adenoma Malignum of the Cervix. A Cancer of deceptively innocent histological pattern. Cancer (1963); 16(5): 549-57.

[11]. Hirai Y, Takeshima N, Haga A, Arai Y, Akiyama F, Hasumi K. A clinicocytopathologic study of adenoma malignum of the uterine cervix.Gynecol Oncol (1998); 70: 219-23.

[12]. Szyfelbein WN, Young RH, Scully RE. Adenoma malignum of the cervix. Cytologic findings. Acta Cytol (1984); 28:691-8.

[13]. Kudo R, Sagae S, Hayakawa O, Kusanagi T, Hashimoto M.The cytological features and DNA content of cervical adenocarcinoma.Diagn Cytopathol (1987); 3:191-7.

[14]. Vogelsang PJ, Nguyen GK, Honore LH. Exfoliative cytology of adenoma malignum (minimal deviation adenocarcinoma) of the uterine cervix. Diagn Cytopathol (1995); 13:146-50.

[15]. Granter SR, Lee KR. Cytologic findings in minimal deviation adenocarcinoma (adenoma malignum) of the cervix. A report of seven cases. Am J Clin Pathol (1996); 105:327-33.

[16]. Young RH, Clement PB. Pseudoneoplastic glandular lesions of the uterine cervix. Semin Diagn Pathol (1991); 8:234-49.

[17]. Yamashita Y, Takahashi M, Katabuchi H, Fukumatsu Y, Miyazaki K, Okamura H. Adenoma malignum: MR appearances mimiking nabothian cysts. Am J Roentogenol(1994); 162: 649-50.

[18]. Utsugi K, Hirai Y, Takeshima N, Akiyama F, Sakurai S, Hasumi K. Utility of the monoclonal antibody HIK-1083 in the diagnosis of adenoma malignum of the uterine cervix.Gynecol Oncol (1999); 75:345-8. 